\title{
Cognitive maps and the hippocampus
}

\author{
PAUL ELLEN \\ Georgia State University, Atlanta, Georgia 30303
}

\begin{abstract}
This paper reviews a number of behavioral issues raised by the O'Keefe-Nadel theory of hippocampus as the substrate of cognitive maps. It was suggested that the mere fact of locomotion between two places was insufficient to assume that the spatial relationship between these two places had been established in a cognitive map. Moreover, it was suggested that locomotor behavior per se is not a necessary precondition for the establishment of a cognitive map. With respect to the effects of cuing, it was shown that when performance on a task unambiguously depends on a cognitive map, cues associated with particular spatial loci are ineffective in guiding performance. Additionally, once a cognitive map is formed, such cues do not alter the map. The role of the hippocampal-fornix system in the development of a cognitive map was supported, although it was shown that hippocampus is not the storage site of the cognitive map.
\end{abstract}

Over the years, a number of investigators (Menzel, 1973; O'Keefe \& Nadel, 1978; Tolman, 1932, 1948; Tolman, Ritchie, \& Kalish, 1946) have suggested that animals can acquire cognitive representations or maps of the geographic environment in which they are located. These maps are assumed to be generated by the animal as it locomotes from one point in space to another: at some subsequent time, the animal exhibits a performance which presumably depends upon the existence of the map. A prototypical example of the role of such mapping is illustrated in the performance of rats on the three-table reasoning problem originally described by Maier (1932b). In this task, the animal is allowed to explore three unbaited tables and the runways connecting them. It is then picked up and placed on one of the tables, where it is fed. Then the animal is removed from that baited table and placed on one of the other tables. The animal's task is to go from the table on which it was just placed to the baited table. On each day, the baited table and start table are randomly varied so that the rat does not have an opportunity to learn a consistent route to the food from day to day. A number of lines of evidence support the view that performance on this task involves the use of a cognitive map of the spatial locus of the tables. If rats are merely fed on one of the tables and then placed on another, they are unable to find food directly on the test trial. Moreover, even if rats are given the test trial over a runway configuration that is different from the one they had covered during their initial exploratory experience, they are able to go directly to the food on the test trial (Herrman, Doherty, \& Ellen, Note 1). Finally, despite being blinded (Maier, 1932 b) or being in a darkened room (Stahl \& Ellen, 1974), the animals are able to go directly to the food on the test trial if they have had the exploratory and feeding experience prior to the experimental manipulation. In short, this task would seem to be an excellent representative of those problem situations in which performance depends on the existence of some kind of cognitive map.

More importantly for the purposes of this workshop, performance on this task has been particularly sensitive to the effects of lesions of either the septal area (Stahl \& Ellen, 1973), fornix (Herrman, Black, Anchel, \& Ellen, 1978), or hippocampus (Rabe \& Haddad, 1969). These findings are of particular importance in view of the recent emphasis (O'Keefe \& Nadel, 1978) on the role of the hippocampus as the neural substrate of cognitive mapping.

The purpose of the present paper is to review some behavioral aspects of the O'Keefe-Nadel theory, with a view towards explicating a number of problems that are implicated in the process of cognitive mapping and that need to be considered in developing a physiological theory of the process. Some of the data we will present here will, in all likelihood, be unfamiliar to most behavioristically oriented investigators, since the data stem from an intellectual tradition not in current vogue. It is the fact that we, as experimenters in 1979, tend to concentrate our attention on the things animals are put through, that is, particular tasks or problems, without regard to the consequences to the animal of these various experiences. Rarely do we address this question or even attempt to determine those aspects of a given task that are, in fact, affecting performance. The behavioral operations we employ, however, are unfortunately not isomorphic with the resultant psychological experiences of the animals. It is, however, these experiences that are particularly critical in dealing with issues such as cognitive maps.

I do not intend to review here all of the details of the O'Keefe-Nadel theory. Their most excellent book presents in considerable detail the pertinent anatomical, physiological, and behavioral data that led to the formulation of the theory. There are, however, several aspects of the theory to which I should like to address myself, as they bear importantly on my concerns here. 
One of the most important aspects of their theory has to do with the role of exploratory behavior in cognitive mapping. According to the theory, such behavior is initiated when there is a mismatch between the hippocampal cognitive map and the perceived environment. This mismatch leads to the firing of "misplace" units (O'Keefe \& Dostrovsky, 1971) which then leads to exploration, and the changed conditions resulting from the exploratory behavior are incorporated into the map. Exploratory behavior functions to reduce the discrepancy between the perceived environment and the cognitive map. Thus, locomotion plays an important role in the process of cognitive mapping. It becomes important, therefore, to examine rather critically the functional role of locomotor behavior in the mapping process and to ask the question as to how spatial relationships get established in cognitive maps. The O'Keefe and Nadel theory assumes that every representation within the map that is generated by movement stands in some relationship to every other representation, regardless of whether the objects generating the various representations have been experienced in either spatial or temporal contiguity. In this section, we will examine the role of locomotion in the construction of a cognitive map and will show that (1) locomotion between two places in space does not necessarily ensure that these places will become spatially related and (2) locomotion may not be a necessary condition at all for establishing such maps.

In the case of cognitive maps, the situation is quite complex. First, it should be noted that a cognitive map, in the case of the rat, is presumably created as the animal locomotes through space (O'Keefe \& Nadel, 1978). The cognitive map would thus arise in a sequential manner, since the animal is in one spatial locus at first and then moves to another. In short, the animal has a series of successive temporal experiences. According to the O'Keefe-Nadel theory, all of the stimuli and objects in the environment are represented in this map, and these representations are systematically related to each other in the form of a cartoon of the environment (Nadel \& O'Keefe, 1974). The theta rhythm codes the sensory inputs into several of the hippocampal lamellae, such that, at the completion of movement, all of the objects and places in the environment are simultaneously related to each other in the cognitive map. The theory thus assumes that all experiences that the animal has had are incorporated into a map where they simply exist, available at any moment for an appropriate input which may activate the representation. Such an input might be a specific distal cue which allows the animal to identify the starting point (i.e., a startbox). Once that has been done, the map then allows the animal to find its way to any desired place. Thus, the existence in experience of both the intended starting and the intended destination points is simply assumed by virtue of these points' having been exposed to the rat at some time during the course of its locomotor behavior. This assumption, however, is quite critical. It is one thing to take for granted the existence of both starting and destination points in a cognitive map; it is another to demonstrate that the various experiences that an animal has had actually give rise to a simultaneous pattern in experience that is necessary for the acquisition of a spatial relationship or a cognitive map. There is no a priori reason for assuming that successive temporal events, as occur when a rat is locomoting from one place to another, become, for the animal, a simultaneous pattern, thereby leading to the formation of a cognitive map with its resulting spatiality. Rather, there is a need to specify the conditions under which successive events $d o$, in fact, give rise to simultaneous patterns in experience.

I should now like to draw attention to a relatively unfamiliar and old experiment which bears on these issues. In 1932, N. R. F. Maier attempted to analyze the experience of a rat when it ran from point $\mathrm{A}$ to point $B$. Assuming that this experience led to the formation of the association A-B, a successive experience, the association $\mathrm{B}-\mathrm{A}$, if established at the same time as the former association, would be a backward association-another temporal experience. If, however, running from A to B produced, instead of a successive experience, one of simultaneity, then B would occupy a certain spatial position relative to $\mathrm{A}$. Thus, the following question can be raised. If the rat learns the position of $B$ relative to $A$, does it at the same time know the position of A relative to $B$ ? Maier (1932a) attempted to address this question in a series of experiments. In the first experiment, three tables, $\mathrm{X}, \mathrm{Y}$, and $\mathrm{Z}$, were placed in a triangular arrangement with interconnecting runways. The rats were then trained to run from $\mathrm{X}$ to $\mathrm{Y}$ for food, from $\mathrm{Y}$ to $\mathrm{Z}$ for food, and finally from $\mathrm{Z}$ to $\mathrm{X}$ for food. This training resulted in the animals' running in a counterclockwise direction around the tables. Following this training, the rats were given food at $\mathrm{X}$ and then placed at $Y$. Would the animal run via a counterclockwise, long route to the table $\mathrm{X}$, or would the animal run directly in a clockwise fashion to the table? It should be noted that the shorter route is the clockwise direct route from $\mathrm{Y}$ to $\mathrm{X}$, while the counterclockwise route is longer, since it requires going to table $\mathrm{Z}$ before going to table $\mathrm{X}$. To the extent that these places exist in the rat's experience as a simultaneous pattern, a cognitive map would be present and the prediction would be that the animals would choose the shorter route to the food. The rats, however, all chose the longer indirect route to the food. This finding suggests that when a rat runs from $\mathrm{X}$ to $\mathrm{Y}$, it does not learn the location of $Y$ relative to $X$. Rather it merely has a temporal experience, which is a successive event. Thus, it cannot simply be assumed, as done by the O'Keefe-Nadel theory, that an animal will acquire a cognitive map of an environment and dis- 
play flexible behavior in a spatial problem simply because it has experienced the various loci in the environment sequentially in the course of its locomotor behavior. Successive experiences do not necessarily give rise to simultaneous patterns in the rat's experience.

Under what circumstances can such an experience as locomoting from $\mathrm{X}$ to $\mathrm{Y}$ give rise to something other than a temporal association of successive events? The experiment just described indicates that the temporal association $\mathrm{X}-\mathrm{Y}$ is not equivalent to the backward running association $\mathrm{Y}-\mathrm{X}$. However, Maier did demonstrate, in a second experiment, that the rat could use the relationship Y-X even though it experienced $\mathrm{X}-\mathrm{Y}$, provided $\mathrm{Y}-\mathrm{X}$ was part of a pattern made up of completely familiar relationships. A rat, in seeking food, could do so in the most efficient manner by running the third leg of the triangle in a direction opposite to what it had been trained on, provided the remaining two legs of the triangle had been experienced in both directions. This observation, when contrasted with the observation that rats could not use a direction opposite to that upon which they had been trained when that direction was not part of a familiar pattern, indicated one of the conditions under which successive experiences give rise to simultaneous patterns. Running two legs of a triangle in both directions increases the rat's knowledge of the third leg despite the fact that the third leg was run in only one direction. Additionally, these findings emphasize the point that the mere exposure to orientation cues from the room is not sufficient to establish spatial relationships between points in the room. In short, it would appear that the fact of locomotion between two geographical loci does not ensure that the representations of these places exist as a simultaneous pattern in experience. The existence of a cognitive map can only be inferred from a specific performance which can be accomplished only if some such map is available, and cannot simply be assumed to have been formed by virtue of the animal's engaging in locomotor exploratory behavior, as is the case in the O'Keefe-Nadel theory.

The view elaborated here implies that in order for successive temporal events to give rise to simultaneous patterns in the rat's experience, these temporal events must or should follow one another fairly closely in time. Otherwise, a simultaneous pattern of representations of objects or places will not arise and there will not be the establishment of spatial relationships.

There is a paucity of data concerning the limits of the time frame within which successive locomotor experiences can give rise to simultaneous patterns. Stahl and Ellen (1974) showed that, on the three-table task, if the rat explored A to B on one day, another combination of places and their connection on a second day, a third combination on the third day, and so forth, this piecemeal, temporally separated exploratory experience was not sufficient to cause the places $\mathrm{A}, \mathrm{B}$, and $\mathrm{C}$ and their interconnections to become spatially connected in the rat's experience, as evidenced by the animal's failure to solve the problem. However, allowing the animal to explore all three places and their interconnections within a single $15-\mathrm{min}$ time period was sufficient for the animal to solve. These observations suggest that not only must the space between points in the geographic environment be traversed in order to become spatially connected, but also the time frame within which the space is traversed is critical. If the space between various points does not get covered within a particular time frame, then these points do not become a simultaneous pattern, that is, spatially connected, and no cognitive map is formed.

Up to now we have suggested that the mere fact of locomotor exploratory behavior is an insufficient basis to infer that a cognitive map with its resultant spatiality has been acquired by an animal. There is still another side of the coin that needs some consideration. Is locomotor behavior even necessary for the formation of a cognitive map? According to the O'Keefe-Nadel theory, such behavior constitutes an important information-processing mechanism whereby the animal acquires spatial information. The postulated role of theta rhythm in the process (this rhythm determines the storage relationships of the various sensory inputs into the hippocampal lamellae) lends further importance to the role of locomotor behavior in the generation of the cognitive map. Thus, the question at hand is whether a cognitive map can be formed when locomotion is excluded. If a spatial representation can be acquired by simply transporting an animal from one place to another, then it would follow that spatiality results when different loci are experienced as simultaneous patterns regardless of how this experience is accomplished-whether by locomotion or by traversing space visually or by being transported.

It will be recalled that Menzel (1973) demonstrated that chimpanzees, which were carried around an outdoor field and shown a number of randomly placed hidden foods, could remember the hiding place and kind of food located in each place. The animals, upon being allowed to retrieve the hidden food, generally ran directly to that food place rather than either retracing the route over which they had been carried or the reverse route. Even more importantly, the chimps did not need to be carried along during the hiding of the food. Findings such as these emphasize the relative unimportance of the locomotor behavior in the formation of a cognitive map. While such behavior may be sufficient, it is not necessary.

Perhaps the strongest support for this view comes from the work of Beritoff (1965) and Ungher and Sirian (1971). These authors have shown that if a blindfolded animal is carried in its cage to a particular area of a room, fed there, and brought back 
to the starting point via the same route, when released and allowed to move freely, the animal would, with its eyes either open or blindfolded, retrace the path to the food. Control procedures eliminated the possibility that this feat was accomplished olfactorily or by means of kinesthetic stimulation of the extremities. Even more complex behaviors were demonstrated in this context. If a blindfolded animal was first carried to a corner of the room where it was fed and then to another corner in the room where it was not fed, the animal, after being released several minutes later, would go directly to the corner where it was fed but not to the other corner. Placing an obstacle in front of the blindfolded animal during the test causes the animal to lose its orientation. However, if the blindfold is removed, the animal can then run directly to the food. In short, a map would seem to have been formed simply by the process of transporting the blindfolded animal. Finally, if a blindfolded animal is taken first to the left for a certain distance without being fed and then to the right where it is fed, it will, upon being released (still with the blindfold on), go directly to the place where it was fed and not along the path used by the experimenter. Of particular importance in this connection are the observations of Ungher and Sirian (1971) with fornix-lesioned animals. The latter are unable to perform these feats of localization.

Finally in this regard, mention should be made of the early work of Gleitman (1955) and McNamara, Long, and Wike (1956), which demonstrated that place learning was possible in the absence of locomotor behavior. In these experiments, rats were required to learn either the locus of food or the locus of shock termination. Merely transporting the animals over the paths in little cars without allowing locomotor activity allowed place learning to occur. Thus, it would appear that place learning or, more generically speaking, cognitive map formation, can occur in the absence of locomotory behavior. Thus, the existing literature describes numerous instances which are not compatible with an important assumption of the O'Keefe-Nadel theory. Two conclusions are possible on the basis of our discussion to this point. The fact of locomotion between two points does not ensure that these points become related in a cognitive map. On the other hand, it is also evident that locomotor behavior itself is not a necessary precondition for the formation of cognitive maps.

Turning now to still another aspect of the O'KeefeNadel theory, we come to the issue of whether place learning involves, as Restle (1957) suggested, nothing more or less than merely a conditioned reaction to distal cues as opposed to proximal cues. In contrast, O'Keefe and Nadel take the relatively strong position that such learning does not depend on the specific stimuli emanating from a particular location or environment but, rather, depends on the existence of a cognitive map that enables the animal to approach by any available route a particular locus in space even though that route had never been utilized before. I suspect part of the problem resides in the fact that most writers generally accept the distal cue hypothesis without examining carefully its implications in any given situation. This point is brought home most forcefully when one compares the O'Keefe, Nadel, Keightley, and Kill (1975) analysis of performance in a circular runway with that of Winson (1978), who used the same runway apparatus to study the ability of hippocampectomized rats to locate food when started from any point in the runway. While O'Keefe et al. argue convincingly that the spatial learning in this problem cannot simply be a reaction to the distal cues of the room, Winson, in the absence of a systematic analysis of the learning, states that such cues are responsible for the learning because "the animal was afforded a full view of the testing room, which contained diverse landmarks"' (1978, p. 160). In essence, instead of testing their effects specifically, Winson simply assumed such cues were operational. The conflict in interpretation would seem to stem from the fact that both Restle's hypothesis and the cognitive mapping hypothesis lead to the same prediction and that the particular test situation lends itself to both hypotheses. In the absence of data, this type of conflict in interpretation will remain unsolved, since the test situation itself is less than unambiguous with respect to this issue.

While we agree with the O'Keefe-Nadel position, we do so on the basis of data in which the role of cognitive mapping is more unambiguously apparent. It will be recalled that performance on the three-table task requires that there have been an exploratory experience prior to the feeding experience in order for a correct choice to be made on the test trial. In the absence of such a prior exploratory experience, rats are simply unable to go consistently to the baited table on the test trial. This finding is not what might be expected from a distal cue hypothesis and very clearly implicates the role of cognitive mapping. Similarly, the work of Maier (1932b) and Stahl and Ellen (1973), using blinded rats or running rats in a darkened room, demonstrated that performance on the three-table task was unimpaired provided the animals had had the prior exploratory experience. Again, the distal cue hypothesis suffers. In short, it would appear that performance on the three-table task is a relatively unambiguous illustration of the role of a cognitive map at work, and distal cues play little or no role in such performance.

Once a cognitive map is involved in performance or if a task unambiguously requires a cognitive map for its successful completion, then, as O'Keefe and Black (1978) indicate, cues in the environment have relatively little effect on the cognitive map. While cues may be necessary for the animal to localize itself, they 
do not function to guide performance when a cognitive map is involved. These points were illustrated in a series of experiments by Herrman, Black, Doherty, and Ellen (1980). In these experiments, we worked with both normal and brain-damaged animals (septal lesions and fornical knife-cut animals). The animals demonstrated the deficit generally seen on the threetable apparatus following these lesions. They were unable to go directly to the food table on the test trial which followed the exploratory and feeding experience. In an attempt to see whether we could alleviate this deficit, a cue light was placed on the food table during the exploratory and feeding experiences and kept there during the test trial. Despite this additional cue, which was visible from the start table during the test trial, neither septal nor fornix-damaged animals were able to solve the problem. Thus, a cue signaling the locus of food did not aid performance in a task in which the utilization of a cognitive map was required. Of particular interest, too, was the observation that when the cue light was placed on the nonbaited table during exploration, feeding, and test trial, the performance of the animals was unchanged. Even the normals were not distracted by the light. The most interesting findings in this study, however, came when the cue light was used only during the feeding and test trials, but not during the preliminary exploration. Under these circumstances the fornical animals, but not the septals, showed an improved performance. Surprisingly, the normals showed a significant decrement in performance. This decrement could be overcome in the case of the normal animals by merely eliminating the exploratory experience. Under the latter conditions, the task is reduced simply to a discrimination task in which the animal is merely required to go to the table containing the light, where they have just been fed. The difference in the response to the cue light by the normal animals as a function of the presence or absence of the exploratory experience emphasizes the view that once a cognitive map is formed, it is not sensitive to cues in the environment (O'Keefe, 1976). Thus, any learning that occurs in response to such cues is not compatible with the learning that occurs during cognitive map formation (Black \& Dalrymple, Note 2) and thus does not get integrated into a solution. Fornix-damaged animals, in contrast, were not influenced in their reactions to the cue light as a function of the presence or absence of the prior exploratory experience. This finding emphasizes the role of the fornix system in the formation of cognitive maps and suggests that, for the fornical animals, the spatial-integrative aspects of the problem were simply not present and that the animals were simply performing as if it were a light discrimination task. In summary, these data emphasize, first, the view that specific cues within a situation cannot compensate for a cognitive map when such is required by the task, and secondly, the relative insensitivity of an already established cognitive map to external cues that happen to be associated with particular places. The consistent fact that fornical or septal lesions interfere with performance on this task reinforces quite strongly the O'Keefe-Nadel concept of the role of the hippocampus in spatial behavior.

There is one final question to which I should like to address myself here. While we are in fundamental agreement with O'Keefe and Nadel with respect to the role of hippocampus in the process of cognitive mapping (we are not committing ourselves to whether we agree with the specific electrophysiological mechanism that they suggest), the question can be posed concerning whether hippocampus is the storage site for such a map. My interpretation of the O'KeefeNadel theory would suggest this to be the case. We have some experiments in progress that bear on this issue. Two groups of rats received ablations of neocortex and underlying hippocampus, as illustrated in Figure 1. The hippocampal damage was limited to the antero-dorsal aspects of the structure. A third group of animals served as controls. Prior to surgery, one group of operates was merely allowed to explore the three-table arrangement for 2 days. They did not, however, receive any feeding or testing during this period. The other operated group did not have this experience. Following surgery, all animals were given the standardized testing regime, which consisted of a 15-min exploratory experience and a 5-min feeding experience, followed by the test trial. The animals were allowed to correct if they made an error on the test trial. Following the test trial and any corrections of errors made on that trial, the animals were given two additional trials in which they could practice running from the start table directly to the food. If, for example, they failed on the test trial but did learn where food was during the correction opportunity, then during these subsequent two trials, they should be able to run directly to the food table. In short, these latter trials merely served to reinforce the learning of the locus of the food on any given day and can be considered to be learning trials. Each day the start and feeding table were varied. As might be expected, the operated animals failed on the first trial. As shown in Table 1,15 of 17 operates failed to reach the $67 \%$ criterion (twice as many correct as incorrect). In contrast, only 3 of 12 normal animals failed to reach the criterion on the first trial. This difference between normals and operates was not unexpected in light of our earlier work. Somewhat surprising, however, were the findings of Table 2, which illustrate the performance on the learning trials. Here a different pattern of results emerges. You will recall that the animals on the learning trials merely had to run back to the table on which they found food during the first trial. As can be seen from Table 2, 7 of the 9 operated rats receiv- 


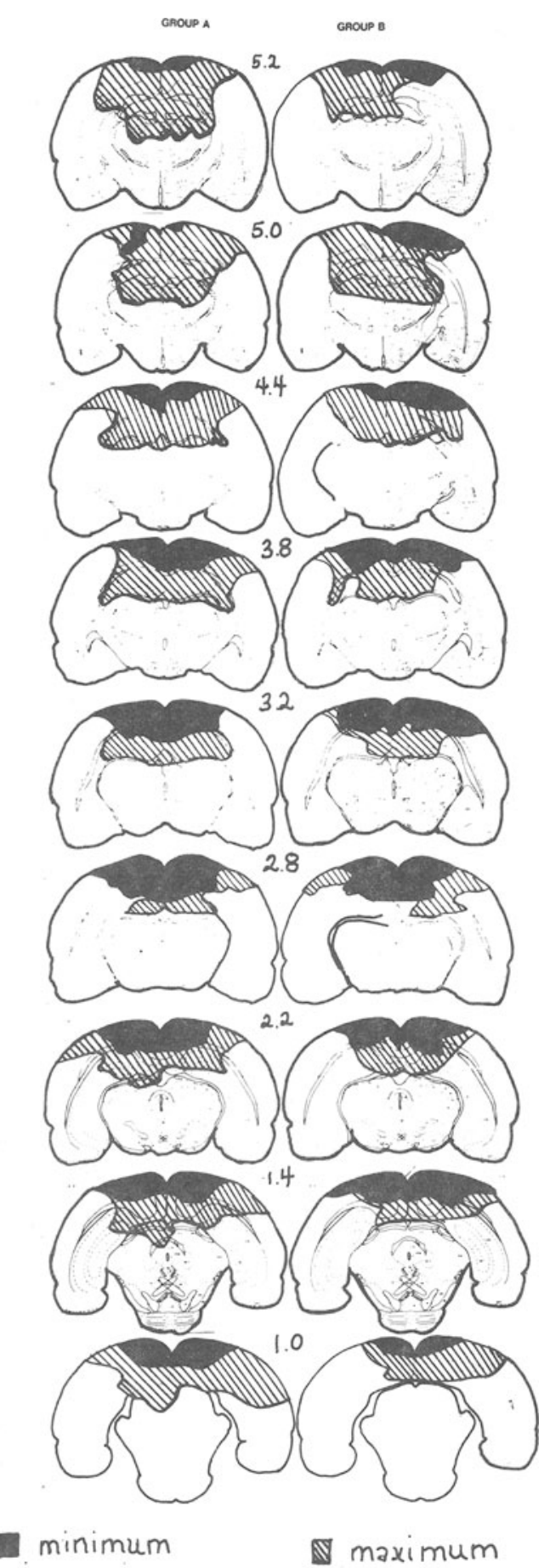

Figure 1. Serial reconstruction of lesions in animals that received prior exploration (Group B) and those that did not (Group A). Minimum and maximum extent of damage illustrated at successive A-P planes of hippocampus.

ing the 2 days of exploration prior to surgery were able to learn the locus of food during the correction procedure. These rats ran directly to the food $70 \%$ of the time on Trial 3. In contrast, none of the animals '
Table 1

Performance on Trial 1

\begin{tabular}{lrrrrr}
\hline & $\begin{array}{c}\text { Number } \\
\text { Solving }\end{array}$ & \multicolumn{2}{c}{$\begin{array}{c}\text { Number } \\
\text { Failing }\end{array}$} & Total \\
\hline Shams & 9 & $(4.2)$ & 3 & $(7.8)$ & 12 \\
Operates (Exploration) & 2 & $(3.4)$ & 7 & $(5.6)$ & 9 \\
Operates (No Exploration) & 0 & $(3.0)$ & 8 & $(5.0)$ & 8 \\
Total & 11 & & 18 & & 29 \\
\hline
\end{tabular}

Note $-\chi^{2}=14.15, p<.01$. Values in parentheses are theoretical frequencies for each cell in the chi-square table.

Table 2

Performance on Trial 3

\begin{tabular}{lrrrrr}
\hline & \multicolumn{2}{c}{$\begin{array}{c}\text { Number } \\
\text { Meeting 67\% } \\
\text { Criterion }\end{array}$} & \multicolumn{2}{c}{$\begin{array}{c}\text { Number } \\
\text { Failing 67\% } \\
\text { Criterion }\end{array}$} & Total \\
\hline Shams & 7 & $(5.8)$ & 5 & $(6.2)$ & 12 \\
Operates (Exploration) & 7 & $(4.3)$ & 2 & $(4.7)$ & 9 \\
Operates (No Exploration) & 0 & $(3.9)$ & 8 & $(4.1)$ & 8 \\
Total & 14 & & 15 & & 29 \\
\hline
\end{tabular}

Note $-\chi^{2}=11.336, p<.01$. Values in parentheses are theoretical frequencies for each cell in the chi-square table.

that had not received the exploration prior to surgery were able to learn the locus of the food. They were able to run directly to the food on the third trial an average of only $50 \%$ of the time. These findings would suggest that once a cognitive map is formed it remains available for spatial learning despite brain damage (Maier, 1938). However, if such damage occurs prior to the formation of a cognitive map, then spatial learning is impossible. Moreover, the fact that such spatial learning occurs following hippocampal damage suggests that the storage site of the cognitive map is not in hippocampus. The hippocampus merely participates in the elaboration of the map. However, in the absence of the hippocampus, mapping does not occur and spatial learning is impaired. Finally, we may consider the detailed nature of the performance of the operated animals when they make errors. On the three-table task, let us assume that the animal is required to run from $\mathrm{A}$ to $\mathrm{B}$ for a correct solution and that this run involves a right turn. Suppose, instead, that the animal runs from $A$ to $\mathrm{C}-\mathrm{a}$ left turn-and makes an error. If the animal is following a turn or response preference, it can get to B from C simply by making another left turn. In other words, if the animal has a turn or response preference, a continued execution of that preference will bring it to the food table. To what extent is the failure to learn the locus of the food by the animals that did not receive the exploratory experience prior to surgery the result of the operation of a turn or response preference when correcting on the first trial? In the case of the animals that did not receive the 2 -day prior exploratory experience, of 67 errors on the first trial, $46(69 \%)$ of the corrections were repetitions of the initially incorrect turn. In contrast, for the animals that did receive the exploratory experience prior to surgery, of 62 first trial 
errors, only $29(47 \%)$ were corrected by a repetition of the first choice turn. In short, animals receiving prior exploration are more likely to correct an error by trying an alternative response or turn when given the opportunity. Thus it would appear that the animals that were given an opportunity to acquire a cognitive map prior to surgery had more behavioral strategies available when an error was made on Trial 1 than did those that were not. The latter apparently could rely only on a turn or response strategy, which, while successful in getting the animal to the food table, did not confer upon it the flexibility necessary to get to the table directly, as measured by Trial 3 performance.

\section{REFERENCE NOTES}

1. Herrman, T., Doherty, D., \& Ellen, P. Places vs. routes in spatial problem solving. Unpublished manuscript.

2. Black, A. H., \& Dalrymple, A. J. Instrumental multistage problem solving in the rat. Unpublished manuscript.

\section{REFERENCES}

Beritoff (Beri rashvili), J. S. [Neural mechanisms of higher vertebrate behavior] (W. T. Liberson, Ed. and transl.). Boston: Little, Brown, 1965.

Gle itman, H. Place learning without prior performance. Journal of Comparative and Physiological Psychology, 1955, 48, 77-79.

Herrman, T., Black, A. H., Anchel, H., \& Ellen, P. Comparison of septal and fornical lesioned rats' performance on the Maier three-table reasoning task. Physiology \& Behavior, 1978, 20, 297-302.

Herrman, T., Black, A. H., Doherty, D., \& Ellen, P. Visual cues fail to alternate deficits on a spatial integration task following septal or fornical damage. Physiological Psychology, 1980, 8, 29-32.

McNamara, H. J., Long, J. B., \& Wike, E. L. Learning without response under two conditions of external cues. Journal of Comparative and Physiological Psychology, 1956, 49, 447-480.

MAIER, N. R. F. A study of orientation in the rat. Journal of Comparative Psychology, 1932, 14, 387-399. (a)

MaIER, N. R. F. Cortical destruction of the posterior part of the brain and its effects on reasoning in rats. Journal of Comparative Neurology, 1932, 56, 179-214. (b)

MaIER, N. R. F. A further analysis of reasoning in rats. III.
The influence of cortical injuries on the process of "direction" in reasoning. Comparative Psychology Monograph, 1938, 15 , 44-85.

Menzel, E. Chimpanzee spatial memory organization. Science, 1973, 182, 943-945.

NADEL, L., \& O'KeEfe, J. The hippocampus in pieces and patches: An essay on modes of explanation in physiological psychology. In R. Bellairs \& E. G. Gray (Eds.), Essays on the nervous system, a Festschrift for Professor J. Z. Young. Oxford: The Clarendon Press, 1974.

O'KeEFE, J. Place units in the hippocampus of the freely moving rat. Experimental Neurology, 1976, 51, 78-109.

O'KeEfe, J., \& Black, A. H. Single unit and lesion experiments on the sensory inputs to the hippocampal cognitive map. In K. Elliot \& J. Whelan (Eds.), Functions of the septohippocampal system. Amsterdam: Elsevier, 1978.

O'KeEfe, J., \& Dostrovsky, J. The hippocampus as a spatial map. Preliminary evidence from unit activity in the freely moving rat. Brain Research, 1971, 34, 171-175.

O'KeEFE, J., \& NADEL, L. The hippocampus as a cognitive map. Oxford: The Clarendon Press, 1978.

O'Keefe, J., Nadel, L., Keightley, S., \& Kill., D. Fornix lesions selectively abolish place learning in the rat. Experimental neurologv, 1975, 48, 152-166.

Rabe, A., Haddad, R. K. Integrative deficit after hippocampal lesions. Proceedings of the 77 th Annual Convention of the American Psychological Association, 1969, 4, 213-214

RESTLE, F. Discrimination of cues in mazes: A resolution of the "place vs. response" question. Psychological Review, 1957, 64, 217-228.

Stahl, J., \& Ellen, P. Septal lesions and reasoning performance in the rat. Journal of Comparative and Phvsiological Psvchology, 1973, 84, 629-638.

Stahl, J., \& Ellen, P. Factors in the reasoning performance of the rat. Journal of Comparative and Physiological Psychology, 1974, 87, 598-604.

Tolman, E. C. Purpose behavior in animals and men. New York: Century, 1932.

Tolman, E. C. Cognitive maps in man and animals. Psychological Review, 1948, 55, 189-208.

Tolman, E. C., Ritchie, B. F., \& Kalish, D. Studies in spatial learning. I. Orientation and the short cut. Journal of Experimental Psychology, 1946, 36, 13-24.

Ungher, J., \& Sirian, S. Vestibular short-term memory deficits due to behavior disturbances induced by fornix lesions in cat. Review of Roumanian Neurology, 1971, 8, 407-417.

Winson, J. Loss of hippocampal theta rhythm results in spatial memory deficit in the rat. Science, 1978, 201, 160-163. 